\title{
Driver Fatigue Detection based on Eye State Analysis
}

\author{
Yong Du ${ }^{1}$ Peijun Ma ${ }^{1}$ Xiaohong Su ${ }^{1}$ Yingjun Zhang ${ }^{1}$ \\ ${ }^{1}$ Department of Computer Science and Technology \\ Harbin Institute of Technology, Harbin, China 150001 \\ duyong@hitsz.edu.cn
}

\begin{abstract}
In this paper, we present an effective vision-based driver fatigue detection method. Firstly, the interframe difference approach binding color information is used to detect face. If exists, the face area is segmented from the image based on a mixed skin tone model. Then we simulate the process of crystallization to obtain the location of eyes within face area. Later, eye area, average height of the pupil and width to height ratio are used to analyze the eye's status. Finally, the driver fatigue is confirmed by analyzing the changes of eye's states. The experimental results show validity of our proposed method.
\end{abstract}

Keywords: driver fatigue detection, face segment, eye location, eye states analysis

\section{Introduction}

The increasing number of traffic accidents due to a diminished driver's vigilance level resulting from sleep deprivation or dull scenes of driving has become a serious problem for society. Statistics show that between $10 \%$ and $20 \%$ of all the traffic accidents are due to drivers with a diminished vigilance level ${ }^{[1]}$. Moreover, accidents related to driver's declined level of vigilance are more serious than other types of accidents, since drowsy drivers often do not take any avoidable actions prior to a collision ${ }^{[2]}$. According to the statistics of National
Highway Traffic Safety Administration (NHTSA), falling asleep while driving causes at least 100,000 automobile crashes annually in the United States and results in more than 40,000 nonfatal injuries and 1,550 fatalities result from these crashes per annum ${ }^{[3][4]}$. With this background, how to supervise the driver's level of vigilance and avoid fatigue driving is essential to the accident prevention.

The rest of the paper is organized as follows: Section 2 provides a brief survey of related researches on driver fatigue detection. In section 3, the outline of our proposed vision-based automatic driver fatigue detection system is presented. Face location method including face existence detection is described in section 4 . Section 5 describes a novel method of the eyes location and eye template generation. Section 6 gives a new driver fatigue analysis method by measuring pupil height, area of the eye and width to height ratio of the eye to identify the eye's states. The experimental results for driver fatigue detection is shown in section 7 . Conclusion and future work are discussed in the last section.

\section{Survey of Previous Work}

In the past decade, many countries have begun to pay great attention to the driver safety problem. Researchers have been working on the detection of driver's drowsiness using various techniques, such as physiological detection ${ }^{[5][6][7]}$,

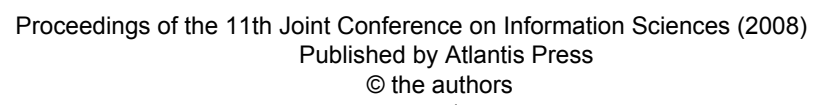


driver behavior monitoring ${ }^{[8]}$, vehicle running status analysis ${ }^{[9]}$ and visionbased detection [10][11][12]][13][14] .

However, among those techniques, vision-based driver fatigue detection method is a natural, non-intrusive and convenient technique to monitor driver's vigilance. When driver fatigue occurs, visual behaviors can be easily observed from changes in their facial features especially from their eyes. It is indicated that the change regularity of eye states have high relativity with the driver's mental states ${ }^{[15]}$. Eyes express the most direct reaction when driver is drowsy or inattention and eye blinking is always used as the basis for driver fatigue detection by researchers.

Zheng pei calculated the ratio of eye closing during a period of time. The ratio can reflect driver's vigilance level ${ }^{[16]}$. Wenhui Dong proposed a method to detect the distance of eyelid, then judged the driver's status by this kind of information ${ }^{[15]}$. Nikolaos P used front view and side view images to precisely locate eyes ${ }^{[10]}$. Edge detection and gray-level projection methods were also applied for the eyes location by Wen-Bing Horng ${ }^{[11]}$. Zutao Zhang located the face by using Haar algorithm and proposed an eye tracking method based on Unscented Kalman Filter ${ }^{[12]}$. Abdelfattah Fawky presented a combination of algorithms, namely wavelets transform, edge detection and $\mathrm{YCrCb}$ transform in the eye detection ${ }^{[13]}$. Qiang Ji depended on IR illumination to locate eyes ${ }^{[14]}$. Eyes always contains two kinds of information: size of opening and duration of the different states. By analyzing the change rules of eyes in fatigue, we propose an efficient approach for driver fatigue detection.

\section{Overall Algorithm}

We present an algorithm of detecting driver fatigue by analyzing the changes of eye's status. The approach contains five phases:

(1) Judgement of face existence

(2) Face location based on mixed skin tone model

(3) Eyes Location and eye template generation

(4) Eye state analysis based on pupil height, area of the eye and width to height ratio

(5) Confirmation of driver's vigilance

The outline of the proposed approach is shown in Fig. 1. In the following sections each step will be discussed in detail.

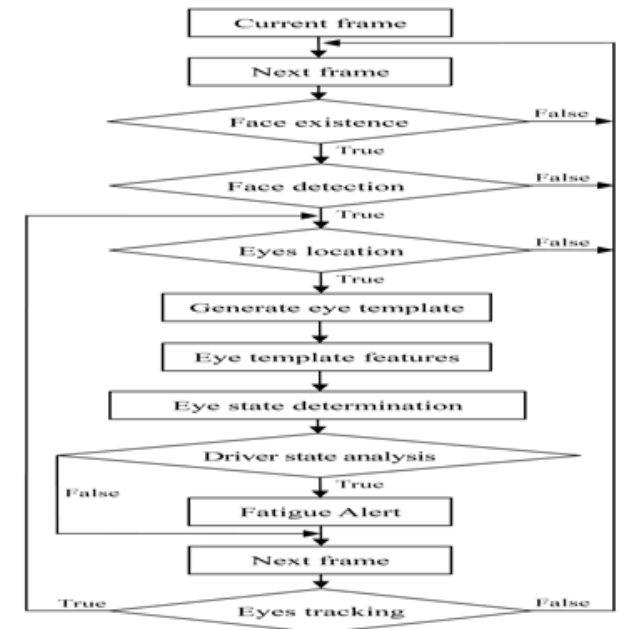

Fig. 1: Flow chart of the approach

\section{Localization of the Face}

When driving on the road, the driver's eyes' position and light condition change constantly. To search and locate the driver's eyes directly in the whole image will not be easy. Moreover, the background is usually complex and unpredictable especially when the automobile is running on the road. So the first step we detect face in order to reduce the range of eye detection, besides, the procedure will

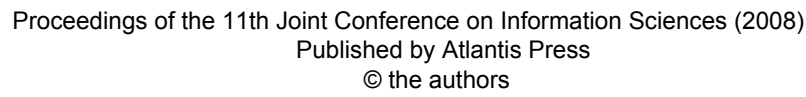


improve the veracity and speed of eyes location and reduce the interference of background.

For purpose of reducing the blindness of face searching, we calculate interframe differences to decide whether there is an moving object. When some object is detected and in the local area there contains skin color information in $\mathrm{YCbCr}$ color space, we believe there is a person before the camera.

Although people of different races distinguish in skin color, the distribution of human skin color in $\mathrm{YCbCr}$ color space can be approximated by a planar Gaussian distribution [15]. Specifically, face area can be segmented from a image by skin color information. To improve the accuracy of detection, in this paper, we use a mixed skin model based on $\mathrm{YCbCr}$ and HSI color Space. Because in the HSI color space hue is independent of brightness, the brightness factor can be excluded from colors. This mixed skin model is more suitable for distinguishing skin and non-skin colors no matter the face is under light or shadowed. The formulas for converting RGB to its corresponding $\mathrm{Cb}, \mathrm{Cr}$ and $\mathrm{H}$ components are given as follows.

$$
H= \begin{cases}\theta & B \leq G \\ 360-\theta & B>G\end{cases}
$$

Where,

$$
\begin{array}{r}
\theta=\arccos \left\{\frac{\frac{1}{2}[(R-G)+(R-B)]}{\left[(R-G)^{2}+(R-G)(G-B)\right]^{1 / 2}}\right\} \\
C b=-0.148 R-0.291 G+0.439 B+128 \\
C r=0.439 R-0.368 G-0.071 B+128
\end{array}
$$

The three components ( $\mathrm{Cb}, \mathrm{Cr}$ and $\mathrm{H}$ ) have different abilities to represent various skin colors and they can be complemented each other. By using this method, it is easy to separate face region from the origin image, as is shown in Fig. 2(b). By performing vertical and horizontal projec- tions on skin pixels, the right, left, top and bottom boundaries of the face can be confirmed if the projection values exceed some threshold which has been set based on experience, as is shown in Fig. 2(c). Usually the normal position of the eyes will be in the upper half part of the face region, as is shown in Fig. 2(d), furthermore, the area is sufficient for us to detect eyes later.

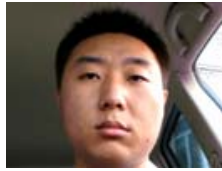

(a) original image

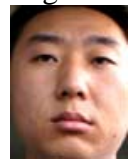

(c) face region

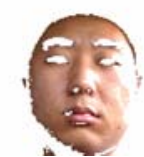

(b) face skin

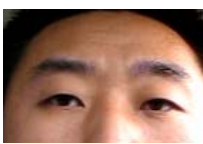

(d) eyes region
Fig. 2: Location result of eyes region

\section{Localization of the Eyes}

According to the thermodynamic principle, for a closed system the minimum of Helmholtz free energy $E_{f}$ is used to describe the equilibrium state in stead of maximum entropy. In such a system, the particles of set $S_{i}$ has the same free energy of $E_{f}^{i}$. Follow the Boltzmann theory and Metropolis rule, the probability of one particle transforming from free energy level $E_{f}^{i}$ to $E_{f}^{j}$ is $p$ which is shown as follows.

$$
p=e^{\frac{-\Delta E_{f}}{k T}}=e^{\frac{-\left|E_{f}^{i}-E_{f}^{j}\right|}{k T}}
$$

where $k$ is a constant and $T$ is thermodynamic temperature.

It is easy to see that the larger the free energy gap between different particles the 
smaller the probability that they can reach the same level. When the free energy gap is same the higher temperature the larger the probability it will be. Consider the theory mentioned above, we take each pixel as various particle and vector norm of properties is defined to represent the free energy $E_{f}$. The free energy of pixel $(\mathrm{m}, \mathrm{n})$ is as follows.

$$
\Delta E_{f}^{(m)}=\sqrt{k_{1}\left(G_{m n}-G^{C}\right)^{2}+k_{2}\left(G_{m n}-G^{C}\right)^{2}+k_{3}\left(H_{m n}-H^{C}\right)^{2}+k_{4}\left(G r y m_{m n}-G r a y^{C}\right)^{2}}
$$

where $C b_{m, n}, C r_{m, n}, H_{m, n}$ and Gray $_{m, n}$ represent the average values of $C b, C r$, $H$ and Gray, which are calculated by pixel (m, n)'s nearest eight neighbors. Similarly, $C b^{C}, C r^{C}, H^{C}$ and Gray ${ }^{C}$ represent the property values of the expected class correspondingly. $k_{1}, k_{2}, k_{3}$ and $k_{4}$ are constants range from 0 to 1 and the four parameters add up to one. Also the gray value can be easily computed by the RGB components.

By considering the diversity to the nearby pixels and the similarity to the eye pixels sufficiently, the specific region of the eyes can be obtained. After this step, projection method is used again to detect the eyes' precise boundaries. The method has been adopted frequently in the eye detection so we needn't describe the details. Our method starts from both sides, left and right, to find eyes, therefore we can detect the eyes separately.

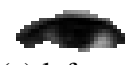

(a) left eye (b) right eye
Fig. 3: Eyes detected

Then we segment the eyes from the image and use them to generate an eye template, by this means we obtain a rather stable eye template for the status analyzing and reduce the influence of light reflections. The eye template is generated as follows.

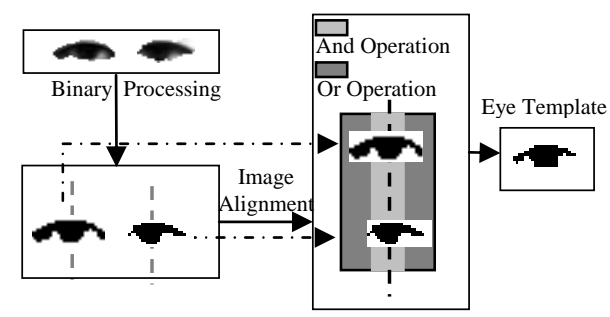

Fig. 4: Eye template generation process

Also the two eyes' positions are recorded, the eyes can be detected in the next frame based on these positions. The searching area expanding 6 pixels in four directions from the eyes' centers in the current frame. If the distance between two eyes detected in the next frame changes greatly, the eye tracking is regarded as failure. Then the face detection and eyes location procedures will be restarted.

\section{Detection of Fatigue}

To distinguish the driver's status the eyes' states should be recognized ahead. There is two factors which can affect the size of the eyes in the frames. On the one hand, human eyes are always different in size. On the other hand, the distance between driver and the camera is the other reason. So we normalize the eye template to a fixed size of $12 \times 30$ before feature extraction. For each eye template, eye area, average height of pupil, width to height ratio are the most important features to judge eye's status which is shown in Table 1.

The eye states can be divided into three types: full open, half open and closed. From the table below we can see that eyes of different states present different features. The experiments also proved that the three indicators (Area, Height and Ratio) are efficient for the eye's states 
recognition. By analyzing the driver's eyes' states changes while driving we discovered two principles which can indicate driver drowsiness. Firstly, if a driver's eyes keep closed over 4 consecutive frames it is believed that the driver is drowsy. Secondly, fatigue can be confirmed if a driver's eyes only change between half open and closed over 8 consecutive frames. Before the system is put into use we trained it in advance to get different states parameters for the driver aiming at improving the accuracy of the driver's status analysis.

Table 1. Eye states and features

\begin{tabular}{c|c|c|c|c|c}
\hline & $\begin{array}{c}\text { Eye } \\
\text { Region }\end{array}$ & $\begin{array}{c}\text { Area } \\
\text { (pixel) }\end{array}$ & $\begin{array}{c}\text { Eye } \\
\text { Template }\end{array}$ & $\begin{array}{c}\text { Average } \\
\text { Height }\end{array}$ & Ratio \\
\hline $\begin{array}{c}\text { Full } \\
\text { Open }\end{array}$ & & 200 & $\mathbf{- 1}$ & 7.6 & 2.8750 \\
\hline $\begin{array}{c}\text { Half } \\
\text { Open }\end{array}$ & & 155 & $\mathbf{-}$ & 6.8 & 3.0000 \\
\hline Closed & $=$ & 114 & & 6.0 & 3.1667 \\
\hline
\end{tabular}

\section{Experimental Results}

From the camera fixed on the car dashboard we acquired the tested videos in the natural driving conditions. The proposed approach has also been tested on the videos. The experimental results are shown as follows.

Table 2. Results of fatigue detection

\begin{tabular}{c|c|c}
\hline & Video 1 & Video 2 \\
\hline Total Frames & 166 & 213 \\
\hline Open eyes & 61 & 68 \\
\hline Half Open Eyes & 82 & 54 \\
\hline Closed Eyes & 23 & 91 \\
\hline False Judgement & 13 & 21 \\
\hline Real Dozing & 3 & 5 \\
\hline Correct Warning & 3 & 5 \\
\hline Tracking Failure & 2 & 0 \\
\hline Precision Rate & $92.17 \%$ & $90.14 \%$ \\
\hline \multicolumn{2}{c}{ Average Precision Rate: $91.16 \%$} \\
\hline
\end{tabular}

The input video format is $320 \times 240$ true color. The average correct rate for eye status recognition can achieve 91.16\%. The different eye states of full open and half open sometimes can not be well distinguished which has caused most false judgements and the fast movement of the driver's head can result in the driver's eyes tracking failure.

The correct warning rate for driver dozing is $100 \%$. Here, a $100 \%$ correct warning rate does not reveal a absolute robustness of the algorithm. If during a period of dozing time the system can detect driver's drowsiness and alarm then we consider this a successful fatigue detection.

\section{Conclusion}

In this paper, we present a new driver fatigue detection method for driving safety. The interframe difference approach is used to decide whether the frame exists a face. Then a mixed skin color model is used to detect face. In the face region we use a novel method by simulating the crystallization process to segment the eyes from the face. By performing projections the eyes can be located precisely. The detected two eyes are used to generate an eye template. We use eye area, average height of the pupil and width to height ratio to distinguish the eye's status. Finally, driver fatigue can be detected based on the rules we discovered. The experimental results show validity of our proposed method for driver fatigue detection under realistic conditions. The future research will focus on obtaining more elaborate eye regions and improving the accuracy of eye states distinction and we also should do more work on detecting the eye's flickering status in the nearly future which can be used to describe the fatigue status more precisely.

\section{References}


[1] L.M. Bergasa, J. Nuevo, M.A. Sotalo, and M. Vazquez, "Real-time system for monitoring driver vigilance,” Proc. IEEE Intelligent Vehicle Symposium, pp. 78-83, 2004.

[2] Qiong Wang, Jingyu Yang, Mingwu Ren, and Yujie Zheng, "Driver Fatigue Detection: A Survey,” Proc. of the 6th World Congress on Intelligent Control and Automation, pp. 85878591, 2006.

[3] D. Royal, "Volume I - Findings report; national survey on distracted and driving attitudes and behaviours, 2002," The Gallup Organization, Washington, D.C., Tech. Rep., DOT HS 809 566, 2003.

[4] Luis M. Bergasa, Jesús Nuevo, "RealTime System for Monitoring Driver Vigilance," IEEE Trans. Intelligent Transportation Systems, vol. 7, no. 1, pp. 63-77, 2006.

[5] Lal, S. K. L., Craig, et al,"Development of an Algorithm for an EEG-based Driver Fatigue Countermeasure," Journal of Safety Research, vol. 34, pp. 321-328, 2003.

[6] Akira Kuramori, Noritaka Koguchi, "Evaluation of Effects of Drivability on Driver Workload by Using Electromyogram,” JSAE Review, vol. 25, pp. 91-98, 2004.

[7] Byung-Chan Chang, Jung-Eun Lim, Hae-Jin Kim, et al, “A Study of Classification of the Level of Sleepiness for the Drowsy Driving Prevention," Proc. SICE Annual Conference, pp. 3084-3089, 2007.

[8] Yoshihiro Takei, and Yoshimi Furukawa, "Estimate of driver's fatigue through steering motion," Proc. IEEE International Conference on Systems,
Man and Cybernetics, vol. 2, pp. 1765-1770, 2005.

[9] Erez Dagan, Ofer Mano, Gideon P. Stein, et al, "Forward Collision Warning with a Single Camera,” Proc. Intelligent Vehicles Symposium, pp. 3742, 2004.

[10] Nikolaos P, "Vision-based Detection of Driver Fatigue," Proc. IEEE Internetional Conference on Intelligent Transportation, 2000.

[11]Wen-Bing Horng, Chih-Yuan Chen, Yi Chang, et al, "Driver Fatigue Detection Based on Eye Tracking and Dynamic Template Matching," Proc. of the 2004 IEEE International Conference on Networking, Sensing \& Control, pp. 7-12, 2004

[12]Zutao Zhang, Jiashu Zhang, “A New Real-Time Eye Tracking for Driver Fatigue Detection," Proc.2006 6th International Conference on ITS Telecommunications, pp8-11,2006.

[13] Abdelfattah Fawky, Sherif Khalil, and Maha Elsabrouty, "Eye Detection to Assist Drowsy Drivers,” IEEE. pp. 131-134, 2007.

[14] Qiang Ji, Zhiwei Zhu, and Peilin Lan, "Real-Time Nonintrusive Monitoring and Prediction of Driver Fatigue," IEEE Transaction on vehicular technology, vol. 53, no. 4, pp. 657-662, 2004.

[15] Wen-Hui Dong, Xiao-Juan Wu, "Driver Fatigue Detection Based on the Distance of Eyelid," Proc. IEEE Int. Workshop VLSI Design \& Video, Tech., pp. 28-30 , 2005.

[16] Zheng Pei, Song Zhenghe, and Zhou Yiming, "Perclos-based recognition algorithms of motor driver fatigue," Journal of China Agricultural University, pp. 104-109, 2004. 